\title{
Triple coronary vessel disease including double vessel chronic total occlusion: Quantitative flow ratio minimizes injury of the single vessel that provides collaterals
}

\author{
Kazuhiro Dan ${ }^{1,2}$, Akira Shinoda ${ }^{1}$, Daichi Tsuzura ${ }^{1}$, Hector M. Garcia-Garcia ${ }^{2}$ \\ ${ }^{1}$ Department of Cardiovascular Medicine, Ichinomiya Nishi Hospital, Aichi, Japan \\ ${ }^{2}$ Section of Interventional Cardiology, MedStar Washington Hospital Center, Washington DC, USA
}

A 67-year-old male with palpitation during physical effort visited the outpatient department. Coronary risk factors were dyslipidemia and smoking. A 12-lead electrocardiogram showed sinus rhythm; $0.5 \mathrm{~mm}$ ST-T depression in leads II, III, aVF, and $\mathrm{V}_{4}-\mathrm{V}_{6}$; and non- $\mathrm{Q}$ wave. Transthoracic echocardiography showed left ventricular ejection fraction of $39 \%$ with anterior hypokinesis and retained wall thickness. Diagnostic coronary angiogram revealed mild stenosis in the left main trunk, severe stenosis in the mid left anterior descending artery (LAD), and chronic total occlusion (CTO) in both of the left circumflex artery and the right coronary artery (Fig. $1 \mathrm{~A}-\mathrm{C}$ ). The LAD was the only vessel supplying collateral coronary blood flow to the other two vessels with CTO, therefore, exercise stress and wire-driven validation for myocardial ischemia could be dangerous due to induced fatal ischemia. Cardiac scintigraphy with drug load did not clearly show ischemia in the LAD (Fig. 1G) and cardiac scintigraphy at rest showed viable myocardium of the whole triple vessel area (Fig. 1H). Functional significance of stenosis at the territory of the LAD was then safely detected in a complex setting by quantitative flow ratio (Fig. 1I-K). SYNTAX score was 48 , however, the patient selected percutaneous coronary intervention (PCI) after discussion with the heart team. Complete revascularization using the bidirectional approach was performed by PCI without complications (Fig. 1D-F), and the ejection fraction improved to $59 \%$. The patient was symptom-free and had no restenosis of all the treated parts by angiographic surveillance at 1 year. The decision making strategy of the present scenario, which has PCI and bypass surgery including several severe settings, is shown (Fig. 1L).

Conflict of interest: None declared

Address for correspondence: Kazuhiro Dan, MD, PhD, Department of Cardiovascular Medicine, Ichinomiya Nishi Hospital, Kaimei-hira 1, Ichinomiya, Aichi, 494-0001, Japan, tel: +81-586-48-0077, Section of Interventional Cardiology, MedStar Washington Hospital Center, 110 Irving St NW, Washington, DC 20010, USA, e-mail: dan27k@gmail.com 


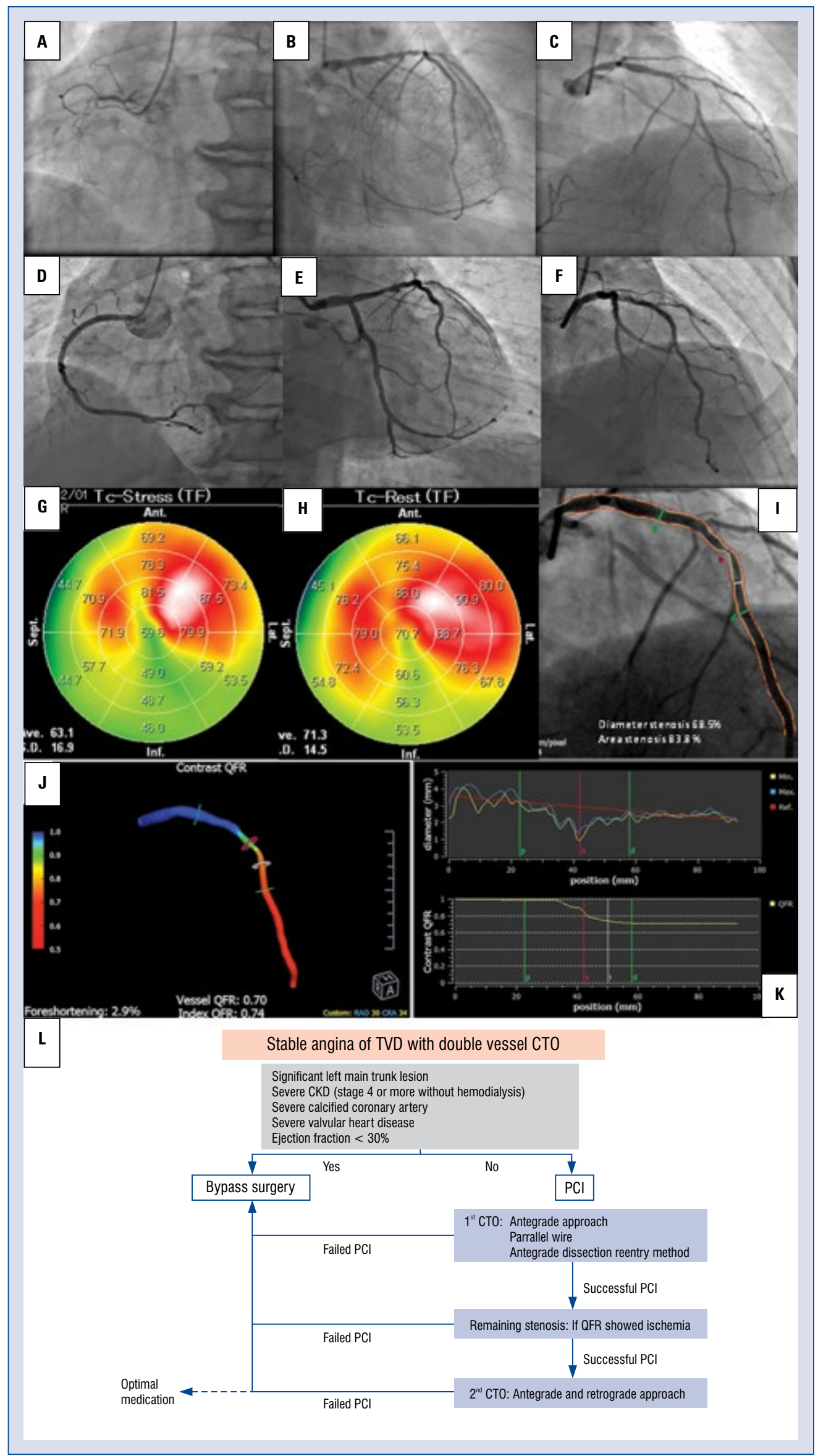


Figure 1. Diagnostic angiographic and physiological images, and final angiographies in percutaneous coronary interventions (PCI). A-C. Diagnostic coronary angiograms; D-F. Final angiographies after PCl. The first $\mathrm{PCl}$ procedure targeted chronic total occlusion (CTO) in the left circumflex artery (LCX) with the antegrade parallel wire technique. The second procedure was for significant stenosis in the mid left anterior descending artery (LAD) with jailed septal branch dilatation for the retrograde approach. The third procedure targeted CTO in the right coronary artery (RCA) with retrograde kissing wire penetration. Finally, the lesions of the left main trunk (LMT) and proximal LCX progressed due to stimulation of devices to the plaque. Cross-over stenting (between the LMT and LCX) and kissing inflation to the LAD with a drug-coated balloon were then performed. The devices were as follows: LCX CTO \#11-13; Guide wire: Wizard $3 g$ (Japan Lifeline, Tokyo, Japan) and Gaia second (Asahi Intecc, Aichi, Japan). Micro catheter: Sasuke (Asahi Intecc). Stent: Xience Alpine (Abbott Vascular, Santa Clara, CA, USA) 2.25*28, 2.5*28. LAD \#7; Xience Alpine 3.0*23. RCA CTO \#1-2; Guide wire: Wizard $3 \mathrm{~g}$ and Gaia second. Micro catheter: Corsair $135 \mathrm{~cm} / 150 \mathrm{~cm}$ (Asahi Intecc). Stent: \#1: Xience Alpine 3.5*28, \#2: Synergy (Boston Scientific, Marlborough, MA, USA) 2.5*28, 2.5*16. LMT \#5-11; Stent: Resolute Integrity (Medtronic, Santa Rosa, CA, USA) $3.5^{*} 22$. Proximal optimization technique $4.5^{*} 8 \mathrm{~mm}$ non-compliant balloon catheter, Powered Lacross 2 (Nipro, Osaka, Japan). Kissing balloon technique to LAD: SeQuent Please (B Braun, Melsungen, Germany) 3.0*20; G, H. Drug-load and rest cardiac scintigraphy with technetium showed mild-moderate ischemia at the territory of RCA and LCX, and viable myocardium of the whole triple vessel area; I-K. A novel functional lesion evaluation by non-invasive wire-free quantitative flow ratio (QFR) by $Q A$ ngio $X A$ 3D (Medis, Leiden, the Netherlands) of the LAD. Area stenosis was $83.8 \%$ by three-dimensional quantitative coronary angiography (3D-QCA), and vessel QFR and lesion QFR values were 0.70 and 0.74 , respectively (cut off value 0.80 ). In QFR analysis, two different angiographic projections $\geq 25^{\circ}$ apart were required with intracoronary nitroglycerine administration prior to angiographic acquisitions. The methodology was to reconstruct anatomical models by 3D-QCA and apply computational fluid dynamics subsequently with the hyperemic flow rate derived by 3D-QCA and Thrombolysis in Myocardial Infarction (TIMI) frame count. QFR assessment showed good agreement and diagnostic accuracy compared with invasive fractional flow reserve (JACC Cardiovasc Interv. 2014; 7: 768-777); L. A strategy in triple coronary vessel disease with double vessel CTO; TVD — triple vessel disease; CKD — chronic kidney disease. 